\title{
A Synthetic Polyester from Plant Oil Feedstock by Functionalizing Polymerization
}

Ye Liu and Stefan Mecking* 
Abstract: Catalytic functionalization/polymerization of castor oil-derived undecenol yields an aliphatic polyester in a single step under mild conditions. The key to selective formation of linear high melting polyester is highly active carbonylation catalysts that at the same time do not undergo strong isomerization.

$T_{\mathrm{h}}$ exclusively from fossil feedstocks. In view of their limited range, alternative renewable sources are desirable in the long term. ${ }^{[1-3]}$ Polyesters are one of the most important classes of polymers, and indeed the more recently developed and commercialized biomass-based polymers are thermoplastic polyesters. ${ }^{[3-6]}$ The preparation of the corresponding monomers can employ a fermentation step, with carbohydrate, most often glucose, as a feedstock. ${ }^{[7]}$ By comparison to fermentation, chemical synthetic routes in which the original molecular structure of the plant biomass employed is substantially retained are attractive as they can be efficient in terms of feedstock utilization and reaction space-time yields, and provide novel properties. Particularly, fatty acids from plant oils can yield longer methylene sequences as a repeat unit motif. ${ }^{[8,9]}$ Targeting polymers, a further functionalization additionally to the carboxy group originating from the fatty acid feedstock is required in order to obtain difunctional repeat units.

To enhance the utilization of renewable feedstocks, straightforward synthetic schemes are desirable. We now report on an efficient functionalization and polymerization in a single step by alkoxycarbonylation of undec-10-ene-1-ol (Scheme 1). The latter is readily available from castor oil by thermal cracking and hydrogenation. ${ }^{[10]}$ However, to date, a functionalizing polymerization requires high pressure $(200 \mathrm{~atm})$ and temperature $\left(160^{\circ} \mathrm{C}\right)$, using $\left[\mathrm{Co}_{2}(\mathrm{CO})_{8}\right]$ as

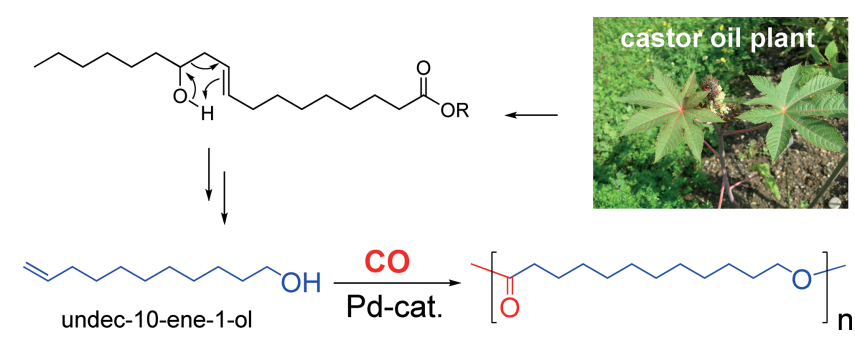

Scheme 1. Carbonylation-polymerization of undecenol derived from castor oil.

[*] Dr. Y. Liu, Prof. Dr. S. Mecking

Chair of Chemical Materials Science, Department of Chemistry, University of Konstanz

Universitätsstraße 10, 78457 Konstanz (Germany)

E-mail: stefan.mecking@uni-konstanz.de

Dr. Y. Liu

State Key Laboratory of Fine Chemicals

Dalian University of Technology

Dalian 116024 (China) a catalyst, and unselectively forms a branched polymer microstructure. ${ }^{[11]}$

Alkoxycarbonylation of olefins is, in principle, a wellstudied reaction. It is used industrially for the production of methyl propionate from ethylene, $\mathrm{CO}$ and methanol. ${ }^{[12,13]}$ Compared to ethylene, substituted olefins are less reactive, but a number of advanced catalyst systems have been reported recently. ${ }^{[14-16]}$ These can even isomerize an internal double bond to selectively form the product ester group at a remote position, for example, methyl oleate can be carbonylated to the terminal linear 1 ,19-diester. ${ }^{[17-19]}$ Notwithstanding this background, the target reaction poses several challenges. Catalysts that are highly active and/or terminal selective in olefin alkoxycarbonylation are also often very active for isomerization. ${ }^{[20,21]}$ For example, the industrially used extremely active catalyst for ethylene methoxycarbonylation with methyl oleate as a substrate affords the aforementioned isomerizing alkoxycarbonylation. With undecenol as a substrate for polymerization, the double bond isomerization can be detrimental when it converts this substrate to undecanal. Formation of this saturated aldehyde not only consumes monomer but also, through acetal formation with two further molecules of substrate, can disturb the stoichiometric balance versus the initial AB-monomer composition.

To probe these anticipated issues, the well-established isomerization active carbonylation catalyst based on the bulky electron rich diphosphine dtbpx was studied in NMR tube experiments. Dissolution of the single component precursor $\left[(\mathrm{dtbpx}) \mathrm{Pd}(\mathrm{OTf})_{2}\right]$ in a $\mathrm{CD}_{2} \mathrm{Cl}_{2} /$ methanol mixture resulted in clean complete conversion to the hydride complex $[(\mathrm{dtbpx}) \mathrm{PdH}(\text { solvent })]^{+}$, in agreement with previous studies $^{[20]}$ (Scheme 2 and Figure 1, bottom. Note that $\mathrm{Pd}-\mathrm{H}$

$\left({ }_{\mathrm{P}^{\prime}}^{\mathrm{P}^{\prime}} \mathrm{Pd}(\mathrm{OTf})_{2}+\mathrm{RCH}_{2} \mathrm{OH} \rightleftharpoons \int_{\mathrm{P}^{\prime}}^{\mathrm{P}^{\prime}{ }^{\mathrm{Pd}^{\prime}} \mathrm{H}^{\mathrm{H}^{+}}} \mathrm{OTf}^{-}+\mathrm{HOTf}+\mathrm{RC}(=\mathrm{O}) \mathrm{H}\right.$

Scheme 2. Suggested formation of the active species from a diphosphine-coordinated Pd" catalyst precursor, accounting for the adverse effect of excess aldehyde. The diphosphine ligand is for example, dtbpx (bis (di-tert-butyl) phosphino-o-xylene).

formation is already observed with 1 equiv $\mathrm{MeOH}$ and is completed with only 10 equivalents). As expected the resulting hydride solution is very active for olefin isomerization, as probed by addition of 1-decene (see Supporting Information, Table S2, entries 13-18). Higher alcohols are known to be less reactive, but also with undecanol instead of methanol the hydride is formed cleanly (Figure 1, center). This suggests that the hydroxyl function of the undecenol monomer is also capable of activating the catalyst precursor to the active hydride species effectively. However, with undecenol as the alcohol component, no hydride species were observed (Figure 1, top). ${ }^{1} \mathrm{H}$ NMR resonances at 9.77 and 4.46 indicate the formation of aldehyde and acetal products (Supporting Information, Figures S1 and S5). The reducing agent in $\mathrm{Pd}-$ hydride formation is considered to be the alcohol present, 


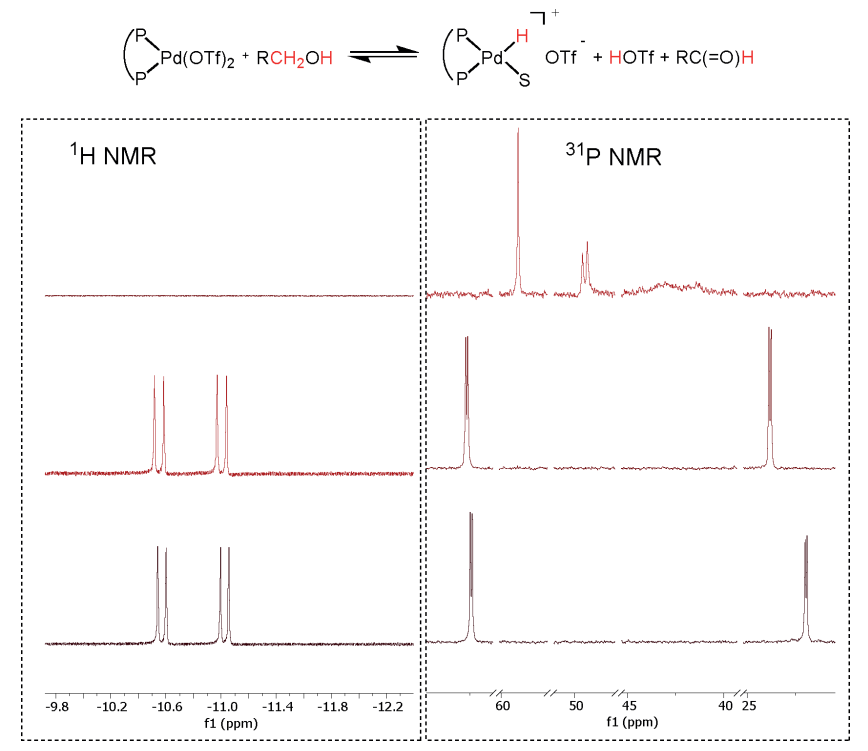

Figure 1. ' $\mathrm{H} N M R$ (left) and ${ }^{31} \mathrm{P}\left\{{ }^{1} \mathrm{H}\right\} \mathrm{NMR}$ (right) spectra of $\mathrm{CD}_{2} \mathrm{Cl}_{2}$ solutions of $\left[(\mathrm{dtbpx}) \mathrm{Pd}(\mathrm{OTf})_{2}\right]$ containing an excess (40 equiv) of methanol (bottom), undecanol (center), and undecenol (top), respectively (room temperature).

even though the expected stoichiometric organic products (aldehydes) have not been confirmed. ${ }^{[22]}$ However, addition of excess nonanal to separately prepared $\mathrm{Pd}-\mathrm{H}$ species results in their consumption (Supporting Information, Figure S2), and the same is expected for undecanal formed by isomerization of the monomer. This NMR scale observation is in accordance with the low efficiency towards carbonylation with dtbpx-based Pd catalysts in pressure reactor experiments (see below). For the case studied herein, the adverse effect of aldehyde can be accounted for by a reversal reaction of the formation for the active species (Scheme 2).

In view of these partly contradictive requirements, a range of diphosphines (Scheme 3) anticipated to vary in their propensity for isomerization and terminal carbonylation were studied for the targeted carbonylation-polymerization of undecenol in pressure reactor experiments (Table 1). Indeed, with dtbpx and related structures (bpx and dtbpp), only small amounts of low molecular weight polyesters are formed, and significant aldehyde formation occurs (entries 1$3)$. By contrast with pytbpx $^{14}$ - known to be extremely reactive in olefin methoxycarbonylation-polyester is formed as the major product (entries 4-6). This is surpassed by xantphos, which converts the undecenol completely to polyester that also has a substantially higher molecular weight of $M_{\mathrm{n}} 1.7 \times 10^{4} \mathrm{~g} \mathrm{~mol}^{-1}$ (entry 9). Note that this value is on the same order as the molecular weights of commercial polyester materials. A molecular weight distribution of $M_{\mathrm{w}} / M_{\mathrm{n}}$ close to 2 indicates a well-behaved nature of the catalytic polycon-

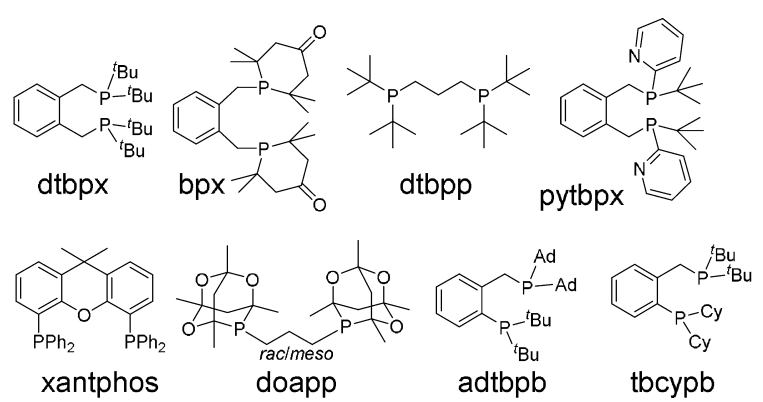

Scheme 3. Diphosphines studied in this work.

Table 1: Carbonylation polymerization of undec-10-en-1-ol. ${ }^{[a]}$

\begin{tabular}{|c|c|c|c|c|c|c|c|c|c|c|c|c|}
\hline \multirow[t]{2}{*}{ Entry } & \multirow{2}{*}{$\begin{array}{l}\text { Diphosphine } \\
\text { ligand }\end{array}$} & \multirow{2}{*}{$\begin{array}{l}T \\
{\left[{ }^{\circ} \mathrm{C}\right]}\end{array}$} & \multirow[t]{2}{*}{$\mathrm{CO}$ [bar] } & \multirow[t]{2}{*}{$t[\mathrm{~h}]$} & \multicolumn{3}{|c|}{ Con. to $[\%]^{[b]}$} & \multirow{2}{*}{$\begin{array}{l}\text { Polyester yield } \\
\text { [g] }\end{array}$} & \multirow{2}{*}{$\begin{array}{l}\text { Linear } \\
\text { Sel. } \\
{[\%]^{[c]}}\end{array}$} & \multirow{2}{*}{$\begin{array}{l}M_{\mathrm{n}} \\
{\left[\mathrm{kg} \mathrm{mol}^{-1}\right]^{[\mathrm{d}]}}\end{array}$} & \multirow{2}{*}{$\begin{array}{l}M_{\mathrm{w}} / \\
M_{\mathrm{n}}[\mathrm{d]}\end{array}$} & \multirow{2}{*}{$\begin{array}{l}T_{\mathrm{m}} \\
{\left[{ }^{\circ} \mathrm{C}\right]^{[e]}}\end{array}$} \\
\hline & & & & & Polyester & $\begin{array}{l}\text { Aldehyde } \\
\text { and } \\
\text { acetal }\end{array}$ & $\begin{array}{l}\text { Isomerized } \\
\text { olefin }\end{array}$ & & & & & \\
\hline 1 & dtbpx & 90 & 30 & 24 & 29 & 24 & 47 & - & - & - & - & - \\
\hline 2 & $b p x$ & 90 & 30 & 24 & 43 & 5 & 53 & $<0.1$ & 98 & 1.7 & 1.2 & - \\
\hline 3 & dtbpp & 90 & 30 & 24 & 39 & 33 & 28 & - & 90 & - & - & - \\
\hline 4 & pytbpx & 90 & 30 & 24 & 90 & 0 & 10 & 1.6 & 89 & 3.3 & 1.5 & 64,70 \\
\hline 5 & pytbpx & 90 & 30 & 72 & 95 & 0 & 5 & 1.7 & 86 & 2.7 & 1.9 & 65,68 \\
\hline 6 & pytbpx & 120 & 30 & 24 & 89 & $<1$ & 11 & 1.3 & 86 & 4.1 & 1.4 & 61,67 \\
\hline 7 & xantphos & 90 & 30 & 24 & 99 & 0 & 2 & 1.7 & 71 & 10.3 & 1.8 & 55,60 \\
\hline 8 & xantphos & 120 & 30 & 24 & $>99$ & 0 & 0 & 1.9 & 71 & 13.6 & 1.8 & 55,63 \\
\hline 9 & xantphos & 120 & 30 & 48 & $>99$ & 0 & 0 & 1.9 & 70 & 17.4 & 1.8 & 54,60 \\
\hline 10 & xantphos & 50 & 30 & 48 & 33 & 0 & 15 & $<0.1$ & - & - & - & - \\
\hline 11 & xantphos & 120 & 20 & 24 & $>99$ & 0 & 1 & 1.9 & 71 & 12.9 & 1.7 & 56,63 \\
\hline 12 & xantphos & 120 & 10 & 24 & 97 & 0 & 3 & 1.6 & 74 & 5.8 & 1.5 & 56,63 \\
\hline 13 & doapp & 90 & 30 & 24 & 47 & 0 & 53 & $<0.1$ & 88 & 1.8 & 1.2 & 51 \\
\hline 14 & adtbpb & 90 & 30 & 24 & 88 & $<1$ & 12 & 1.5 & 98 & 3.3 & 1.4 & 73,75 \\
\hline 15 & adtbpb & 90 & 10 & 24 & 82 & 3 & 15 & 1.0 & 98 & 3.1 & 1.3 & 68,71 \\
\hline 16 & tbcypb & 90 & 30 & 24 & 68 & 10 & 22 & 0.9 & 89 & 1.22 & 1.9 & 50,55 \\
\hline
\end{tabular}

[a] Reaction conditions: $\mathrm{PdOAc}_{2}$ /diphosphine/MSA/olefin =1/2/10/500, molar ratio, $1.9 \mathrm{~g}$ undec-10-en-1-ol in $4.0 \mathrm{~mL}$ toluene. [b] Determined by ${ }^{1} \mathrm{H}$ NMR. [c] Determined from integration of ${ }^{13} \mathrm{C}$ NMR carbonyl resonances. [d] Determined by GPC in THF, calibrated with polystyrene standards. [e] Determined by DSC. 
densation reaction. Differences in the melting points (Table 1) of the polyesters obtained with different diphosphines can be related to their linearities. Branched units that disturb crystallization, resulting from carbonylation of a secondary alkyl, are mainly present in the form of methyl branches (Figures 2, and S8-S11, S25, S27 in the Supporting Information). Notably, with the mixed-substituted benzyl backbone adtbpb, formation of virtually entirely linear polyester was possible. Hereby, a peak melting point of $T_{\mathrm{m}}$ $75^{\circ} \mathrm{C}$ could be achieved (Table 1 , entries 14 and 15, Figure S29 in the Supporting Information). This matches with a linear polyester sample prepared by traditional polycondensation of 12-hydroxydodecanoic acid for comparison. ${ }^{[23]}$

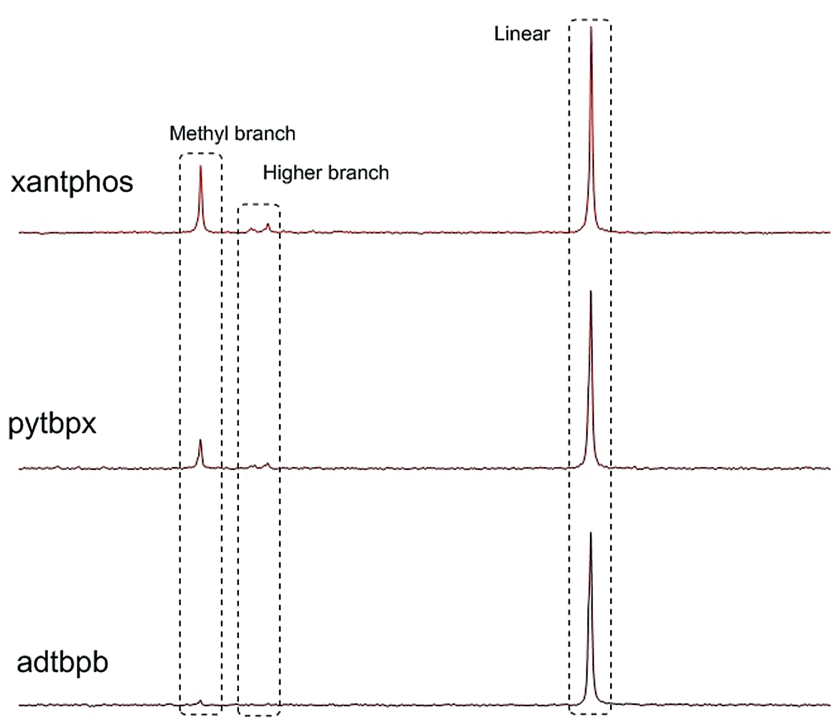

$\begin{array}{llllllllllllll}178.0 & 177.5 & 177.0 & 176.5 & 176.0 & 175.5 & 175.0 & 174.5 & 174.0 & 173.5 & 173.0 & 172.5\end{array}$ f1 (ppm)

Figure 2. ${ }^{13} \mathrm{C}$ NMR carbonyl resonances of polyesters obtained with different diphosphine ligands: adtbpb (bottom; Table 1, entry 14), pytbpx (center; Table 1, entry 6); and xantphos (top; Table 1, entry 9).

Note that a high isomerizing ability (problematic in this carbonylation-polymerization) and a high linear selectivity (desirable) are not contradictive (see Table 1, entry 2 with bpx), but in alkoxcarbonylation of olefins in general often go hand in hand, as selectivity depends on the preference for $\mathrm{CO}$ insertion into the terminal alkyl compared to branched alkyls.

End group analysis of the polyesters by TOSCY and DOSY NMR spectroscopy and MALDI-TOF spectrometry (Supporting Information, Figures S12-S16) reveals terminal $-\mathrm{OH}$ and internal olefin end groups. Molecular weights from NMR spectroscopy agree reasonably well with molecular weights determined by GPC, considering that GPC compared to polystyrene standards usually overestimates molecular weights for hydrocarbon backbone polymers. A considerable deviation is found for the higher molecular weight polyesters formed with xantphos. This is due to cycle formation, as evident from MALDI-TOF (Supporting Information, Figure S17). It is well established that in polycondensations a considerable portion of cycles are formed when high conversions are achieved, and the concentration of reacting groups is very low which favors intramolecular cyclization. ${ }^{[24,25]}$

In view of these findings, the isomerization behavior of selected catalysts was studied further by NMR spectroscopy, under conditions similar to pressure reactor polymerizations (but in the absence of $\mathrm{CO}$ ). In all cases, isomerization of the terminal double bond of the undecenol substrate occurred even at room temperature or slightly above, and at $90{ }^{\circ} \mathrm{C}$ no terminal olefin remained. However, only with dtbpx and adtbpb (though less rapid) full isomerization across the entire $\mathrm{C}_{11}$ chain to form the aldehyde occurred, while this was not the case for xantphos and pytbpx (Supporting Information, Figure S6). The identified microstructures of polyesters formed with different diphosphine ligands are confirmed by selectivities observed in alkoxycarbonylation of model olefins (1- and 2-octene) with undecanol (Supporting Information, Figure S18).

In summary, carbonylation-polymerization of undecenol can yield polyesters at moderate conditions, namely 10 to $20 \mathrm{~atm}$ of $\mathrm{CO}$ pressure with virtually full conversion of the substrate to the desired polyester. In general, any polycondensation requires sufficient reaction rates even at low concentrations of the reacting groups, as present in the final stages of the reaction, to achieve reasonably high molecular weights. It is notable that the catalytic carbonylations studied herein can fulfill this criterion. The key to achieving these high conversions is the use of catalysts that are very active for carbonylation, which at the same time do not promote extensive isomerization. The latter results in loss of monomer and catalyst deactivation by the formed aldehyde. Despite these constraints, even high linearities and consequently higher melting points can be achieved with appropriately chosen catalysts. The case of adtbpb shows that in achieving this a certain propensity for isomerization can be tolerated, providing that carbonylation is competitive with isomerization. This can also serve as a guideline for further development of this chemistry.

\section{Experimental Section}

Carbonylation-polymerization was carried out in a $20 \mathrm{~mL}$ stainless steel reactor equipped with a glass inlet and a magnetic stirring bar, heated in a metal block. The reactor was purged repeatedly with nitrogen. Undec-10-en-1-ol, $\mathrm{Pd}(\mathrm{OAc})_{2}$, additional diphosphine ligand, methanesulfonic acid (MSA), and toluene were weighed in a Schlenk tube equipped with a magnetic stir bar in a glovebox. The mixture was stirred vigorously and cannula-transferred into the reactor under a nitrogen stream. A static pressure of 20-30 bar CO was applied, and the reactor was heated to the desired temperatures under stirring. After the designated reaction time, the reactor was cooled to room temperature and the $\mathrm{CO}$ pressure was released slowly. An aliquot was taken out and $\mathrm{CDCl}_{3}$ was added for NMR spectroscopy analysis to determine conversion and the product distribution. The reactor and glass inlet were washed with dichloromethane, and for the combined phases, the solvents were removed in vacuum. To completely remove any unreacted monomer which could disturb further analysis, the remaining crude polymer was repeatedly redissolved in dichloromethane, precipitated with methanol, isolated by filtration, and washed several times with methanol. The collected 
polymer was dried in vacuum at $50^{\circ} \mathrm{C}$ prior to determination of the yield by weighing and NMR, GPC, and DSC analysis.

\section{Acknowledgements}

Y.L. is grateful to the Alexander von Humboldt Foundation for a postdoctoral research fellowship. The authors thank Kaiwu Dong from Matthias Beller's group for providing pytbpx ligand and Lars Bolk for GPC and DSC measurements.

\section{Conflict of interest}

The authors declare no conflict of interest.

Keywords: atom economy · carbonylation .

homogeneous catalysis · polymerization - sustainable polymers

[1] X. Zhang, M. Fevre, G. O. Jones, R. M. Waymouth, Chem. Rev. 2018, 118, 839-885.

[2] Y. Zhu, C. Romain, C. K. Williams, Nature 2016, 540, 354-362.

[3] S. Mecking, Angew. Chem. Int. Ed. 2004, 43, 1078-1085; Angew. Chem. 2004, 116, 1096-1104.

[4] "Biodegradable Polymers": A. Künkel, J. Becker, L. Börger, J. Hamprecht, S. Koltzenburg, R. Loos, M. B. Schick, K. Schlegel, C. Sinkel, G. Skupin, M. Yamamoto, in Ullmann's Encylopedia of Industrial Chemistry, Wiley-VCH, Weinheim, 2016.

[5] I. Delidovich, P. J. C. Hausoul, L. Deng, R. Pfützenreuter, M Rose, R. Palkovits, Chem. Rev. 2016, 116, 1540-1599.

[6] C. Vilela, A. F. Sousa, A. C. Fonseca, A. C. Serra, J. F. J. Coelho, C. S. R. Freire, A. J. D. Silvestre, Polym. Chem. 2014, 5, 3119 3141.

[7] G.-Q. Chen, M. K. Patel, Chem. Rev. 2012, 112, 2082-2099.
[8] U. Biermann, U. Bornscheuer, M. A. R. Meier, J. O. Metzger, H. J. Schäfer, Angew. Chem. Int. Ed. 2011, 50, 3854-3871; Angew. Chem. 2011, 123, 3938-3956.

[9] F. Stempfle, P. Ortmann, S. Mecking, Chem. Rev. 2016, 116, $4597-4641$.

[10] F. C. Naughton, J. Am. Oil Chem. Soc. 1974, 51, 65-71.

[11] D. Quinzler, S. Mecking, Chem. Commun. 2009, 5400-5402.

[12] W. Clegg, G. R. Eastham, M. R. J. Elsegood, R. P. Tooze, X. L. Wang, K. Whiston, Chem. Commun. 1999, 1877-1878.

[13] B. Harris, Ingenia 2010, 45, 18-23.

[14] K. Dong, X. Fang, S. Gülak, R. Franke, A. Spannenberg, H. Neumann, R. Jackstell, M. Beller, Nat. Commun. 2017, 8, 14117.

[15] J. D. Nobbs, C. H. Low, L. P. Stubbs, C. Wang, E. Drent, M. van Meurs, Organometallics 2017, 36, 391-398.

[16] J. T. Christl, P. Roesle, F. Stempfle, G. Müller, L. Caporaso, L. Cavallo, S. Mecking, ChemSusChem 2014, 7, 3491-3495.

[17] C. Jiménez-Rodriguez, G. R. Eastham, D. J. Cole-Hamilton, Inorg. Chem. Commun. 2005, 8, 878-881.

[18] D. Quinzler, S. Mecking, Angew. Chem. Int. Ed. 2010, 49, 43064308; Angew. Chem. 2010, 122, 4402-4404.

[19] V. Goldbach, P. Roesle, S. Mecking, ACS Catal. 2015, 5, 59515972.

[20] P. Roesle, C. J. Dürr, H. M. Möller, L. Cavallo, L. Caporaso, S. Mecking, J. Am. Chem. Soc. 2012, 134, 17696-17703.

[21] P. Roesle, L. Caporaso, M. Schnitte, V. Goldbach, L. Cavallo, S. Mecking, J. Am. Chem. Soc. 2014, 136, 16871-16881.

[22] W. Clegg, G. R. Eastham, M. J. Elsegood, B. T. Heaton, J. A. Iggo, R. P. Tooze, R. Whyman, S. Zacchini, Organometallics 2002, 21, $1832-1840$.

[23] L. van der Mee, F. Helmich, R. de Bruijn, J. M. Vekemans, A. R. Palmans, E. W. Meijer, Macromolecules 2006, 39, 5021-5027.

[24] H. R. Kricheldorf, A. Lorenc, J. Spickermann, M. Maskos, J. Polym. Sci. Part A 1999, 37, 3861-3870.

[25] H. R. Kricheldorf, D. Langanke, J. Spickermann, M. Schmidt, Macromolecules 1999, 32, 3559-3564. 